\title{
Does elevated intra-abdominal pressure during laparoscopic colorectal surgery cause acute gastrointestinal injury?
}

\author{
Zhenghao Cai ${ }^{1}$, Manu L.N.G. Malbrain ${ }^{2}$, Jing Sun ${ }^{1}$, Ruijun Pan ${ }^{1}$, Junjun Ma ${ }^{1}$, Bo Feng ${ }^{1}$, Feng Dong ${ }^{1}$, Minhua Zheng ${ }^{1}$ \\ ${ }^{1}$ Ruijin Hospital, Shanghai Jiao Tong University School of Medicine, Shanghai, China \\ ZZiekenhuis Netwerk Antwerpen, ZNA Stuivenberg, Antwerpen, Belgium
}

Videosurgery Miniinv 2015; 10 (2): 161-169

DOI: $10.5114 /$ wiitm.2015.52210

\begin{abstract}
Introduction: The incidence of acute gastrointestinal injury (AGI) after colorectal surgery is low when laparoscopic techniques are used. While elevated intra-abdominal pressure (IAP) and intra-abdominal hypertension (IAH) are associated with AGI grade II, little is known about the relation between increased IAP during laparoscopy and subsequent AGl.

Aim: To assess the impact of increased IAP during laparoscopic colorectal surgery on the incidence of postoperative AGI. Material and methods: Sixty-six patients (41 men and 25 women) with colorectal cancer undergoing elective laparoscopic colorectal surgery were randomized into 3 groups, according to different IAP levels during $\mathrm{CO}_{2}$ pneumoperitoneum $(10 \mathrm{~mm} \mathrm{Hg}, 12 \mathrm{~mm} \mathrm{Hg}$ and $15 \mathrm{~mm} \mathrm{Hg})$. We recorded the incidence of AGl after surgery by assessing the following parameters: time to first flatus/defecation, time to first bowel movement, time to tolerance of semi-liquid food and the occurrence of vomiting/diarrhea. Moreover, inflammatory mediators were measured before the induction of $\mathrm{CO}_{2}$ pneumoperitoneum and on postoperative day 1.

Results: Acute gastrointestinal injury occurred in 15 (27.3\%) patients. In all 3 study groups, the elevation of IAP during $\mathrm{CO}_{2}$ pneumoperitoneum did not significantly increase the occurrence of symptoms of AGl, vomiting or diarrhea. Lower IAP levels did not significantly accelerate recovery of gastrointestinal function or shorten postoperative hospital stay. The changes in serum IL-6 after surgery did not correlate with the value of IAP.

Conclusions: The level of IAP elevation during laparoscopic colorectal surgery does not increase the occurrence of AGl after surgery.
\end{abstract}

Key words: laparoscopic surgery, intra-abdominal pressure, colorectal carcinoma, acute gastrointestinal injury, postoperative recovery, inflammatory mediators.

\section{Introduction}

Acute gastrointestinal injury (AGI) was proposed by the Working Group on Abdominal Problems (WGAP) of the European Society of Intensive Care Medicine (ESICM) in 2012 [1]. According to the severity, a grading system of AGI was established for clinical and research purposes [1]. Similar symptoms of gastrointestinal dysfunction, including ileus, diar- rhea and vomiting, can be observed in postoperative patients after abdominal surgery [2]. These symptoms are AGI grade I according to the ESICM WGAP recommendations [1].

Though technical improvements of laparoscopic colorectal surgery and perioperative care in recent years [3-5] have significantly reduced the incidence of AGI correlated symptoms, resulting in improved clinical recovery after colorectal surgery [6-8], con- 
tinuous insufflation of $\mathrm{CO}_{2}$ into the peritoneal cavity still results in elevated intra-abdominal pressure (IAP) for several hours during surgery. Traditionally insufflation pressures are limited to an IAP of $15 \mathrm{~mm} \mathrm{Hg}$ and are generally between 12 and $15 \mathrm{~mm} \mathrm{Hg}$, corresponding with intra-abdominal hypertension ( $\mathrm{IAH})$ grade I [9]. Furthermore, this level of $\mathrm{IAH}$ has been associated with AGI grade II, which means gastrointestinal dysfunction more severe than might be expected in relation to standard abdominal procedures and surgery [1]. Moreover, in analogy to other intra-abdominal organs (like the liver, kidneys and spleen), the gut also suffers from a reduction of blood flow during IAH. A splanchnic ischemia-reperfusion (IR) injury is induced by the insufflation and desufflation of $\mathrm{CO}_{2}$ pneumoperitoneum while IAP increases up to $12 \mathrm{~mm} \mathrm{Hg}[10,11]$, with postoperative AGI correlated symptoms observed in animal models of IR injury [12].

Most clinical trials on this regard has not examined the IAP level during $\mathrm{CO}_{2}$ pneumoperitoneum. Moreover, Kronberg et al. did not introduce IAP into their novel predictive score evaluating possible risk factors for postoperative ileus (POI) after laparoscopic colorectal surgery [13]. Kozlik et al. had indicated the development of oxidative stress performed at high pressure pneumoperitoneum in a clinical study, but they focused mainly on the duration of laparoscopy, not on the intra-abdominal pressure [14]. Therefore, it remained to be discussed whether the level of IAP during laparoscopic surgery was related to the incidence of postoperative AGI. If this is the case, then the IAP induced by $\mathrm{CO}_{2}$ pneumoperitoneum during laparoscopic surgery should be set below a critical threshold in the future.

In addition, POI, a common symptom of AGI, is considered to be a major determining factor related to increased hospital stay and increased recovery time after surgery $[15,16]$. Various pathogenic mechanisms have been suggested in relation to POI, including inflammatory mediators such as interleukin-6 (IL-6) and tumor necrosis factor $\alpha$ (TNF- $\alpha$ ) [17]. These cytokine levels have been reported to increase in patients with high IAP induced by $\mathrm{CO}_{2}$ pneumoperitoneum during laparoscopic surgery, and IL-6 and TNF- $\alpha$ levels may be elevated for more than 1 day [18]. In this study we compared the degree of increase of these cytokines caused by different levels of IAP. We hypothesized that IAP levels and cytokine levels correlate with the incidence of POI.

\section{Aim}

The aim of the study was to assess the impact of increased IAP during laparoscopic $\mathrm{CO}_{2}$ pneumoperitoneum on the incidence of postoperative AGI. To verify the safety of laparoscopic colorectal surgery performed with IAP up to $15 \mathrm{~mm} \mathrm{Hg}$.

\section{Material and methods}

\section{Ethics}

The Ethics Committee of Ruijin Hospital, Shanghai Jiao Tong University School of Medicine, Shanghai, China, approved the protocol of this study. The project was registered at the Chinese Clinical Trial Registry (Registration Number: ChiCTR-TRC-13003292) in accordance with the ethical standards of the 1964 Declaration of Helsinki and its later amendments. Out of 84 consecutive patients who met the study inclusion criteria, 66 (78.6\%) agreed to participate after signed informed consent was obtained.

\section{Sample size calculation}

The standardized formula $N=\pi_{0}\left(1-\pi_{0}\right)(Z \alpha+Z \beta)^{2} /$ $\delta^{2}$ was used to calculate the sample size $(\alpha=0.05$, $1-\beta=0.2, Z \alpha=1.6449, Z \beta=1.2816 ; \pi_{0}$ refers to the population rate of endpoint; $\delta$ refers to the expected significant difference of sample rate). In a preliminary experiment before this study, we observed 13 eligible patients who met the inclusion criteria within 30 days. Five (38.5\%) patients presented AGI correlated symptoms. On the other hand, a reduction of incidence of AGI by $50 \%$ was considered clinically significant. Thus, $\pi_{0}=38.5 \%, \delta=\pi_{0} / 2$. The theoretical sample size $(N)$ hence is 54.76 . With a loss to follow-up probability of about 0.2 , the actual sample size was calculated at $(1+0.2) \times N=65.71 \approx 66$ in this study.

\section{Patients and eligibility}

Patients from Shanghai Minimally Invasive Surgery Center, Shanghai, China were enrolled for this study. Inclusion criteria were as follows: age between 40 and 80 years; American Society of Anesthesiologists (ASA) score I or II; a biopsy proven histological diagnosis of colorectal carcinoma; no clinical evidence of metastasis; undergoing laparoscopic colorectal surgery. Exclusion criteria were: contraindication of laparoscopic surgery (e.g. extensive intra-abdominal adhesion); emergency procedure; evidence of bowel ileus/obstruction before 
surgery; unresectable mass; a planned stoma (e.g abdominoperineal resection of rectal carcinoma, protective ileal stoma); an unexpected stoma; conversion to open surgery; short-term re-operation; postoperative opioid analgesic usage; or persistent uncorrected severe fluid and electrolyte imbalance (e.g. hypokalemia, hypomagnesemia) [19].

\section{Study design and intervention}

The eligible patients were randomized into 3 groups using Microsoft Excel software (Redmond, WA, USA), in which the IAP of $\mathrm{CO}_{2}$ pneumoperitoneum during laparoscopic surgery was set at three levels in different groups: group 1: $10 \mathrm{~mm} \mathrm{Hg}$; group 2: $12 \mathrm{~mm} \mathrm{Hg}$; and group 3: $15 \mathrm{~mm} \mathrm{Hg}$. The fixed IAP was set and monitored via the $\mathrm{CO}_{2}$ pneumoperitoneum insufflation system (STORZ Thermoflator, KARL STORZ GmbH \& Co. KG, Tuttlingen, Germany) by an anesthesiologist.

The laparoscopic procedures were standardized and performed by an identical surgical team in all groups. Right hemicolectomy was performed in a supine position with the head of the bed raised $30^{\circ}$, while sigmoid colectomy and anterior resection of the rectum were performed in a modified lithotomy position (head-down tilt). Left hemicolectomy required an alteration between these two positions. A small incision with a varying size corresponding to the resection specimen was performed in order to accomplish the anastomosis. The nasogastric tube (NG tube) was inserted before the anesthesia induction and was removed immediately when the patients regained consciousness in order to avoid anesthetic accidents (inhalation of vomit).

\section{Blinding}

A double-blind method was adopted in this study. Patients, operating surgeon or surgeons taking care of patients were not informed about the IAP level applied during the surgery; only the anesthesiologist was aware, keeping the IAP level fixed during laparoscopy.

\section{End-points and outcomes}

The first flatus/defecation, the first bowel movement (the sense of bowel sound before flatus/defecation) and the occurrence of vomiting/diarrhea were self-reported by the patient and recorded in the study files. Patients were visited at least once daily after surgery by an experienced surgeon evaluating clinical recovery, including the tolerance of semi-liquid food, other postoperative intra-abdominal complications such as anastomotic leak, surgical site oozing, etc, and finally the discharge time. The anesthesiologist recorded the duration of surgery and $\mathrm{CO}_{2}$ pneumoperitoneum and the intra-operative blood loss. At the onset of the $\mathrm{CO}_{2}$ pneumoperitoneum and at 6 a.m. on postoperative day 1 (POD1), serum IL- 6 and TNF- $\alpha$ levels were measured.

Criteria for allowing semi-liquid food were: normothermia; no adverse reactions with liquid food; normal defecation/free-flow of stoma. Discharge criteria included: tolerance of semi-liquid food for more than $24 \mathrm{~h}$; normothermia; normal defecation/ free-flow of stoma.

Postoperative ileus was defined as absence of flatus/defecation or bowel movement before POD2 according to Vather et al. [2]. The AGI was considered to occur in patients who presented: nausea/ vomiting unrelated to anesthetic reaction; diarrhea (three or more loose or liquid stools per day) for more than 1 day; POI.

\section{Statistical analysis}

The data collection was done using EpiData 3.1 software (freeware available at www.epidata.dk, Odense, Denmark), while all statistical analysis was performed with the IBM Statistical Package for the Social Sciences (SPSS 13.0, Chicago, IL, USA). Continuous data were expressed by mean $\pm S D$, and intergroup differences were determined by one-way analysis of variance (ANOVA) analyses (represented as $\bar{x} \pm \mathrm{s}$ ). Categorical data were expressed as frequency distributions and/or percentages, and the $\chi^{2}$ test or Fisher's exact test was used to determine intergroup differences. For other variables, a non-parametric test was used (represented as median and quartiles). Two-sided $p$ values $<0.05$ were considered to indicate statistical significance.

\section{Results}

\section{Patient demographics}

All 66 enrolled patients were analyzed, and each group consisted of randomly assigned 22 patients. No statistically significant differences in baseline characteristics were noted between patients in the 3 groups (Table I). Tumor location, type of surgery, duration of surgery, duration of $\mathrm{CO}_{2}$ pneumoperito- 
Table I. Patient demographics and characteristics (all patients enrolled)

\begin{tabular}{|c|c|c|c|c|}
\hline Baseline characteristic & $\begin{array}{c}\text { Group } 1 \\
(\mathrm{IAP}=10 \mathrm{~mm} \mathrm{Hg}) \\
n=22\end{array}$ & $\begin{array}{c}\text { Group } 2 \\
(\mathrm{IAP}=12 \mathrm{~mm} \mathrm{Hg}) \\
n=22\end{array}$ & $\begin{array}{c}\text { Group } 3 \\
(\mathrm{IAP}=15 \mathrm{~mm} \mathrm{Hg}) \\
n=22\end{array}$ & Value of $p$ \\
\hline Age $(\bar{x} \pm s)$ & $61.9 \pm 8.7$ & $64.3 \pm 11.7$ & $64.7 \pm 6.4$ & $0.476^{\#}$ \\
\hline Gender ratio (male/female) & $14 / 8$ & $13 / 9$ & $14 / 8$ & $0.938^{*}$ \\
\hline Tumor location, $n(\%)$ : & & & & $0.146^{\ddagger}$ \\
\hline Ascending colon & $2(9.1)$ & $6(27.3)$ & $7(31.8)$ & \\
\hline Transverse colon & $0(0)$ & $3(13.6)$ & $0(0)$ & \\
\hline Descending colon & $1(4.5)$ & $1(4.5)$ & $1(4.5)$ & \\
\hline Sigmoid colon & $11(50)$ & $7(31.8)$ & $11(50)$ & \\
\hline Rectum & $8(36.4)$ & $5(22.7)$ & $3(13.6)$ & \\
\hline Type of surgery, $n(\%):$ & & & & $0.088^{\ddagger}$ \\
\hline Right hemicolectomy & $2(9.1)$ & $9(40.9)$ & $7(31.8)$ & \\
\hline Left hemicolectomy & $2(9.1)$ & $1(4.5)$ & $1(4.5)$ & \\
\hline Sigmoid colectomy & $0(0)$ & $0(0)$ & $3(13.6)$ & \\
\hline Anterior resection of rectum & $15(68.2)$ & $11(50)$ & $9(40.9)$ & \\
\hline Stoma or subtotal colectomy & $3(13.6)$ & $1(4.5)$ & $2(9.1)$ & \\
\hline Operation time $[\mathrm{h}]:$ & & & & $0.453^{\S}$ \\
\hline Median (quartile) & $1.6(1.4-2.0)$ & $1.8(1.6-2.2)$ & $1.9(1.5-2.2)$ & \\
\hline Pneumoperitoneum time [h]: & & & & $0.185^{\S}$ \\
\hline Median (quartile) & $0.9(0.8-1.1)$ & $1.1(0.9-1.3)$ & $1.0(0.8-1.3)$ & \\
\hline Intra-operative blood loss [ml]: & & & & $0.562^{\S}$ \\
\hline Median (quartile) & $100(50-163)$ & $80(50-120)$ & $100(50-100)$ & \\
\hline $\begin{array}{l}\text { Removal of NG tube within } 6 \mathrm{~h} \\
\text { after surgery, } n(\%)\end{array}$ & $22(100)$ & $21(95.5)$ & $18(81.8)$ & $0.117^{\ddagger}$ \\
\hline AJCC staging, $n(\%)$ : & & & & $0.437^{\ddagger}$ \\
\hline I & $7(31.8)$ & $5(22.7)$ & $9(40.9)$ & \\
\hline$\|$ & $6(27.3)$ & $8(36.4)$ & $5(22.7)$ & \\
\hline III & $8(36.4)$ & $4(18.2)$ & $6(27.3)$ & \\
\hline IV & $1(4.5)$ & $5(22.7)$ & $2(9.1)$ & \\
\hline \multicolumn{2}{|c|}{ Postoperative intra-abdominal complications: } & & & $0.171^{\ddagger}$ \\
\hline None & $22(100)$ & $21(95.5)$ & $19(86.4)$ & \\
\hline Chylous fistula & 0 & $1(4.5)$ & 0 & \\
\hline Anastomotic leak & 0 & 0 & $1(4.5)$ & \\
\hline
\end{tabular}

${ }^{\#}$ Analysis of variance (ANOVA) test - Welsh's test, ${ }^{\star}$ Pearson's $\chi^{2}$ test, ${ }^{\ddagger} \chi{ }^{2}$ test - Fisher's exact test, ${ }^{\S}$ nonparametric test - Kruskal Wallis' test. 
neum, intra-operative blood loss, and removal of NG tube within $6 \mathrm{~h}$ after surgery were not significantly different between the 3 groups (Table I). The postoperative anatomopathologic staging (based on the TNM classification system, 2010, $7^{\text {th }}$ edition) was also not statistically significantly different $(p=0.44)$. The length of follow-up ranged from 7 to 14 days after surgery and $4(6.1 \%)$ patients presented with early postoperative intra-abdominal complications other than AGI: one chylous fistula, one anastomotic leak and two cases of surgical site oozing. However, we could not determine a statistical correlation between IAP and early postoperative intra-abdominal complications other than AGI $(p=0.17)$.

\section{Postoperative AGI incidence}

Of the 66 enrolled patients, 11 patients were excluded from the AGI analysis (respectively 3, 3 and 5 in groups 1, 2 and 3). The reasons for exclusion were as follows: 4 patients underwent an unexpected stoma; the other 6 received opioid analgesia after surgery; and finally in 1 patient from group 1 , the surgeon changed the IAP level (from 10 to $15 \mathrm{~mm} \mathrm{Hg}$ ) during surgery.

Of the 55 remaining patients, 15 (27.3\%) patients presented with postoperative AGI correlative symptoms (respectively 6,3 and 6 in groups 1,2 and 3), of which 8 cases were regarded as AGI grade I and 7 cases were classified as AGI grade II. Neither the incidence nor the grade of AGI showed a statistically significant difference between the 3 groups ( $p=0.41$, and $p=0.36$ respectively). Recovery of gastrointestinal function was not significantly improved in patients receiving a low IAP level (group 1) compared with those having received higher IAP levels (groups 2 and 3) during laparoscopy. The level of IAP elevation was not related to increased occurrence of AGI symptoms, neither POI $(p=0.92)$ nor diarrhea $(p=0.67)$. Postoperative hospital stay was similar in the 3 groups $(p=0.27)$ (Table II).

Compared with the 55 patients included in the AGI analysis, the use of opioid analgesia and the application of the stoma did not alter the time to the first flatus/defecation, the time to the first bowel movement or the post-operative hospital stay (Table III). However, patients with a protective ileal stoma achieved earlier tolerance of semi-liquid food than others ( $p<0.001$, Mann-Whitney U-test).

\section{Serum cytokine levels}

The patients in the 3 groups had equal serum IL-6 and TNF- $\alpha$ levels at the onset of $\mathrm{CO}_{2}$ pneumoperitoneum (Table IV). At 6 a.m. on POD 1, serum IL-6 levels were increased ( $p<0.001$, Wilcoxon's test) while TNF- $\alpha$ levels remained virtually unchanged compared to the pre-operative levels ( $p=0.17$, Wilcoxon's test). However, the increase in IL- 6 levels was not significantly different in the 3 groups $(p=0.27)$ (Table IV). The 11 POI cases did not have a significantly increase in IL-6 levels compared to other patients ( $p=0.98$, Mann-Whitney U-test).

\section{Discussion}

For the past 20 years, $\mathrm{CO}_{2}$ pneumoperitoneum during laparoscopic surgery has been considered to compromise cardiopulmonary functions due to the increased IAP during the procedure [20]. Hemodynamic changes such as a decrease in cardiac index, an increase in mean arterial blood pressure (MAP), systemic vascular resistance (SVR) and central venous pressure (CVP) have been observed previously, while alterations in respiratory function include reduced compliance and a transient reduction in lung volumes and capacities [20-22]. Increased IAP may result in neuroendocrine alterations with an increase in plasma concentration of renin, aldosterone, cortisol, adrenalin and noradrenalin in various degrees [20-22].

However, little is known about acute gastrointestinal dysfunction caused by $\mathrm{CO}_{2}$ pneumoperitoneum during laparoscopic surgery. Possible reasons include the variety of definitions and classification of GI dysfunction [23], the lack of reliable biomarkers for assessing GI function [24], and the underestimated GI injury in post-laparoscopic patients [25, 26].

The consensus and recommendations about acute gastrointestinal injury (AGI) [1] allowed us to investigate these issues. The gastrointestinal symptoms of AGI, including nausea/vomiting [27], diarrhea (three or more loose or liquid stools per day) $[28,29]$, paralysis of the lower GI tract (paralytic ileus) [15], and abnormal bowel sound [30] can be observed frequently in post-operative patients undergoing laparoscopic colorectal surgery. Thus far, there has been no published study indicating whether the elevated IAP during laparoscopic surgery (generally between 12 and $15 \mathrm{~mm} \mathrm{Hg}$ ) is associated with postoperative AGI or is related to increased occurrence of the above-mentioned Gl symptoms. 
Table II. The AGI correlated parameters (only valid patients included)

\begin{tabular}{|c|c|c|c|c|}
\hline AGI parameters & $\begin{array}{c}\text { Group } 1 \\
n=19\end{array}$ & $\begin{array}{c}\text { Group } 2 \\
n=19\end{array}$ & $\begin{array}{c}\text { Group } 3 \\
n=17\end{array}$ & Value of $p$ \\
\hline AGI incidence, $n(\%)$ : & $6(31.6)$ & $3(15.8)$ & $6(35.3)$ & $0.413^{\ddagger}$ \\
\hline AGl grade, $n(\%)$ : & & & & $0.361^{\S}$ \\
\hline None & $13(68.4)$ & $16(84.2)$ & $11(65.7)$ & \\
\hline Grade I & $2(10.5)$ & $2(10.5)$ & $4(23.5)$ & \\
\hline Grade II & $4(21.1)$ & $1(5.3)$ & $2(11.8)$ & \\
\hline POI incidence, $n(\%)$ : & $4(21.1)$ & $3(15.8)$ & $4(23.5)$ & $0.915^{\ddagger}$ \\
\hline Time to first bowel movement [h]: & & & & $0.209^{\#}$ \\
\hline Average $\pm \mathrm{SD}(\bar{x} \pm \mathrm{s})$ & $36.9 \pm 24.6$ & $25.9 \pm 16.4$ & $32.9 \pm 12.3$ & \\
\hline Time to first flatus/defecation [h]: & & & & $0.545^{\S}$ \\
\hline Median (quartile) & $31.3(16.8-45.6)$ & $40.8(20.9-64.5)$ & $31.9(20.8-51.0)$ & \\
\hline Time to semi-liquid food [h]: & & & & $0.531^{\S}$ \\
\hline Median (quartile) & $142.3(118.9-144.9)$ & $137.4(118.7-143.4)$ & $139.5(119.1-145.5)$ & \\
\hline Days with vomiting, $n(\%)$ : & & & & $1.000^{\S}$ \\
\hline 0 & $18(94.7)$ & $18(94.7)$ & $16(94.1)$ & \\
\hline 1 & $1(5.3)$ & $1(5.3)$ & $1(5.9)$ & \\
\hline Days with diarrhea, $n(\%)$ : & & & & $0.670^{\S}$ \\
\hline 0 & $14(73.7)$ & $16(84.2)$ & $13(76.5)$ & \\
\hline 1 & $4(21.1)$ & $3(15.8)$ & $3(17.6)$ & \\
\hline 2 & 0 & 0 & $1(5.9)$ & \\
\hline 3 or more & $1(5.3)$ & 0 & 0 & \\
\hline Post-operative hospital stay [days]: & & & & $0.638^{\S}$ \\
\hline Median (quartile) & $8(8-9)$ & $8(8-9)$ & $8(8-9.5)$ & \\
\hline
\end{tabular}

"Analysis of variance (ANOVA) test - Welsh's test, ${ }^{\ddagger} \chi^{2}$ test - Fisher's exact test, ${ }^{\S}$ Nonparametric test - Kruskal-Wallis test.

Our present prospective randomized controlled study indicates no significant correlation between the level of elevated intra-abdominal pressure during surgery and the incidence of postoperative AGI. Out of the total 66 enrolled patients, 11 (16.7\%) were excluded from the AGI analysis in accordance with the exclusion criteria, which corresponded to the loss to follow-up probability of about $20 \%$. The incidence of $\mathrm{AGI}$ was $35.3 \%$ in group 3 (IAP $=15 \mathrm{~mm} \mathrm{Hg}$ ), similar to the result of the preliminary experiment (38.5\%). The standard deviation (SD) of the other end-points in this study was $18.9 \mathrm{~h}$ (time to the first bowel movement), $21.6 \mathrm{~h}$ (time to the first flatus/defection) and $18.8 \mathrm{~h}$ (time to tolerance of semi-liquid food) respectively, which was in line with the results of Muller et al. [31].

The strengths of the present study are as follows. First, a blinded surgeon was appointed to visit the patients after surgery and to decide the discharge time, limiting bias. The operating surgeon was also blinded with regard to the level of IAP applied during laparoscopy to avoid bias. Second, the serum inflammatory mediators were measured with the same testing method and the same lab machine. Third, in order to avoid a confounding bias, opioid analgesia was avoided if other analgesics were sufficiently effective (protocol for postoperative analgesics: level I: paracetamol; level II: nefopam, phloroglucinol; level III: 
Table III. Analysis of excluded patients

\begin{tabular}{|c|c|c|c|c|}
\hline Parameters & $\begin{array}{l}\text { Valid patients } \\
\qquad n=55\end{array}$ & $\begin{array}{l}\text { Opioid analgesia } \\
\qquad n=4\end{array}$ & $\begin{array}{c}\text { Stoma } \\
n=6\end{array}$ & Value of $p$ \\
\hline Time until bowel movement [h]: & & & & $0.652^{*}$ \\
\hline Average $\pm \mathrm{SD}(\bar{x} \pm \mathrm{S})$ & $31.9 \pm 18.9$ & $34.1 \pm 22.0$ & $23.4 \pm 10.5$ & \\
\hline Time until flatus/defecation [h]: & & & & $0.256^{\star}$ \\
\hline Average $\pm S D(\bar{x} \pm s)$ & $36.8 \pm 21.6$ & $46.0 \pm 18.3$ & $23.4 \pm 10.5$ & \\
\hline Time until semi-liquid food [h]: & & & & $0.004^{\S}$ \\
\hline Median (quartile) & $138.8(118.9-144.3)$ & $130.3(111.8-144.6)$ & $81.3(67.4-92.6)$ & \\
\hline Post-operative hospital stay [days]: & & & & $0.638^{\S}$ \\
\hline Median (quartile) & $8(8-9)$ & $8.5(8-9)$ & $8(8-8.5)$ & \\
\hline
\end{tabular}

${ }^{*}$ Analysis of variance (ANOVA) test, ${ }^{\S}$ nonparametric test - Kruskal-Wallis test.

Table IV. Serum cytokine levels (only valid patients included)

\begin{tabular}{|lllcc|}
\hline Cytokine & $\begin{array}{c}\text { Group 1 } \\
n=19\end{array}$ & $\begin{array}{c}\text { Group 2 } \\
n=19\end{array}$ & $\begin{array}{c}\text { Group 3 } \\
n=17\end{array}$ & Value of $p$ \\
\hline \begin{tabular}{l} 
Pre-operative IL-6 [ng/l]: \\
\hline Median (quartile)
\end{tabular} & $2.0(2.0-2.7)$ & $2.8(2.0-5.9)$ & $2.1(2.0-3.0)$ & $0.080^{\S}$ \\
\hline \begin{tabular}{l} 
Post-operative IL-6 [ng/l]: \\
\hline Median (quartile)
\end{tabular} & $10.7(7.5-17.7)$ & $11.3(5.4-14.5)$ & $9.4(6.7-18.2)$ & $0.808^{\S}$ \\
\hline \begin{tabular}{l} 
Pre-operative TNF [ng/l]: \\
\hline Median (quartile)
\end{tabular} & $9.5(7.4-10.7)$ & $9.4(8.5-13.4)$ & $8.5(7.8-9.5)$ & $0.185^{\S}$ \\
\hline \begin{tabular}{l} 
Post-operative TNF [ng/l]: \\
\hline Median (quartile)
\end{tabular} & $9.0(8.5-12.5)$ & $10.3(7.8-12.0)$ & $8.2(6.4-12.2)$ & $0.272^{\S}$ \\
\hline \begin{tabular}{l} 
Delta IL-6 [ng/l]: \\
\hline Median (quartile)
\end{tabular} & & & & $0.266^{\S}$ \\
\hline
\end{tabular}

tramadol or subcutaneous morphine). Fourth, fluid and electrolyte imbalances were adjusted as soon as possible, especially hypokalemia and hypomagnesemia, as these have been reported to aggravate bowel ileus [18]. Finally, other confounding factors such as the duration of surgery and the duration of $\mathrm{CO}_{2}$ pneumoperitoneum were similar among the three groups.

The limitations of our study are as follows: First, the lack of objective serum biomarkers for Gl function/dysfunction does not permit a quantitative integrative analysis. We could only compare the occurrence and recovery of Gl symptoms separately. Second, the incidence of AGI in our study was low, so that statistically significant differences may not be detected due to a small sample size and possibly low statistical power. Third, although some possible confounding factors, such as type of surgery, duration of surgery and $\mathrm{CO}_{2}$ pneumoperitoneum, and removal of the NG tube within $6 \mathrm{~h}$ after surgery, were equally balanced between the three study groups, the results could have been more convincing if we had done a stratified analysis on these factors in a larger sample size. Fourth, the IAP was not measured postoperatively, to check whether a postoperative IAP increase was related to AGI.

With regard to inflammatory mediators that play a role in POI, such as IL-6 and TNF- $\alpha[17,32]$, previous studies have reported increased serum levels after laparoscopic surgery [18, 33-35]. The elevated 
IAP in this study did not attenuate the immune response, but the test frequency was restricted. Previous studies have shown a dose-response effect in relation to IAP elevation and serum cytokine levels. Hence dynamic monitoring of these cytokines would have provided more information reflecting the real postoperative immune status.

\section{Conclusions}

The incidence of postoperative AGI in patients undergoing laparoscopic colorectal surgery is not correlated with the level of elevated intra-abdominal pressure during $\mathrm{CO}_{2}$ pneumoperitoneum. These "negative" results need to be validated in a multi-centre randomized controlled trial with a larger sample size.

\section{Conflict of interest}

The authors declare no conflict of interest.

\section{References}

1. Reintam Blaser A, Malbrain ML, Starkopf J, et al. Gastrointestinal function in intensive care patients: terminology, definitions and management. Recommendations of the ESICM Working Group on Abdominal Problems. Intensive Care Med 2012; 38: 384-94.

2. Vather R, Trivedi S, Bissett I. Defining postoperative ileus: results of a systematic review and global survey. J Gastrointest Surg 2013; 17: 962-72.

3. Wilmore DW, Kehlet H. Management of patients in fast track surgery. BMJ 2001; 322: 473-6.

4. Kehlet H, Wilmore DW. Multimodal strategies to improve surgical outcome. Am J Surg 2002; 183: 630-41.

5. Kehlet H, Wilmore DW. Evidence-based surgical care and the evolution of fast-track surgery. Ann Surg 2008; 248: 189-98.

6. Chen HH, Wexner SD, Iroatulam AJ, et al. Laparoscopic colectomy compares favorably with colectomy by laparotomy for reduction of postoperative ileus. Dis Colon Rectum 2000; 43: 61-5.

7. Kang CY, Chaudhry OO, Halabi WJ, et al. Outcomes of laparoscopic colorectal surgery: data from the Nationwide Inpatient Sample 2009. Am J Surg 2012; 204: 952-7.

8. van Bree SH, Vlug MS, Bemelman WA, et al. Faster recovery of gastrointestinal transit after laparoscopy and fast-track care in patients undergoing colonic surgery. Gastroenterology 2011; 141: 872-80.

9. Kirkpatrick AW, Roberts DJ, De Waele J, et al. Intra-abdominal hypertension and the abdominal compartment syndrome: updated consensus definitions and clinical practice guidelines from the World Society of the Abdominal Compartment Syndrome. Intensive Care Med 2013; 39: 1190-206.

10. Nickkholgh A, Barro-Bejarano M, Liang R, et al. Signs of reperfusion injury following $\mathrm{CO} 2$ pneumoperitoneum: an in vivo microscopy study. Surg Endosc 2008; 22: 122-8.
11. Eleftheriadis E, Kotzampassi K, Papanotas K, et al. Gut ischemia, oxidative stress, and bacterial translocation in elevated abdominal pressure in rats. World I Surg 1996; 20: 11-6.

12. Moore-Olufemi SD, Kozar RA, Moore FA, et al. Ischemic preconditioning protects against gut dysfunction and mucosal injury after ischemia/reperfusion injury. Shock 2005; 23: 258-63.

13. Kronberg U, Kiran RP, Soliman MS, et al. A characterization of factors determining postoperative ileus after laparoscopic colectomy enables the generation of a novel predictive score. Ann Surg 2011; 253: 78-81

14. Koźlik J, Przybyłowska J, Mikrut K, et al. Selected oxidative stress markers in gynecological laparoscopy. Videosurgery Miniinv 2015; 10: 92-100.

15. Story SK, Chamberlain RS. A comprehensive review of evidence-based strategies to prevent and treat postoperative ileus. Dig Surg 2009; 26: 265-75.

16. Lubawski J, Saclarides T. Postoperative ileus: strategies for reduction. Ther Clin Risk Manag 2008; 4: 913-7.

17. Behm B, Stollman N. Postoperative ileus: etiologies and interventions. Clin Gastroenterol Hepatol 2003; 1: 71-80.

18. Han C, Ding Z, Fan J, et al. Comparison of the stress response in patients undergoing gynecological laparoscopic surgery using carbon dioxide pneumoperitoneum or abdominal wall-lifting methods. J Laparoendosc Adv Surg Tech A 2012; 22: 330-5.

19. Carroll J, Alavi K. Pathogenesis and management of postoperative ileus. Clin Colon Rectal Surg 2009; 22: 47-50.

20. Wahba RW, Beique F, Kleiman SJ. Cardiopulmonary function and laparoscopic cholecystectomy. Can J Anaesth 1995; 42: 51-63.

21. O'Leary E, Hubbard K, Tormey W, et al. Laparoscopic cholecystectomy: haemodynamic and neuroendocrine responses after pneumoperitoneum and changes in position. $\mathrm{Br} J$ Anaesth 1996; 76: 640-4.

22. Andersson L, Lindberg G, Bringman S, et al. Pneumoperitoneum versus abdominal wall lift: effects on central haemodynamics and intrathoracic pressure during laparoscopic cholecystectomy. Acta Anaesthesiol Scand 2003; 47: 838-46.

23. Rombeau JL, Takala J. Summary of round table conference: gut dysfunction in critical illness. Intesive Care Med 1997; 23: 476-9.

24. Piton G, Manzon C, Cypriani B, et al. Acute intestinal failure in critically ill patients: is plasma citrulline the right marker? Intesive Care Med 2011; 37: 911-7.

25. van der Voort M, Heijnsdijk EA, Gouma DJ. Bowel injury as a complication of laparoscopy. Br J Surg 2004; 91: 1253-8.

26. Bishoff JT, Allaf ME, Kirkels W, et al. Laparoscopic bowel injury: incidence and clinical presentation. J Urol 1999; 161: 887-90.

27. Fujii Y. The utility of antiemetics in the prevention and treatment of postoperative nausea and vomiting in patients scheduled for laparoscopic cholecystectomy. Curr Pharm Des 2005; 11: 3173-83.

28. Wiesen P, Van Gossum A, Preiser JC. Diarrhoea in the critically ill. Curr Opin Crit Care 2006; 12: 149-54.

29. Hassel DA, Smith PA, Nieto JE, et al. Di-tri-octahedral smectite for the prevention of post-operative diarrhea in equids with surgical disease of the large intestine: results of a randomized clinical trial. Vet J 2009; 182: 210-4.

30. Baid H. A critical review of auscultating bowel sounds. Br I Nurs 2009; 18: 1125-9. 
31. Muller SA, Rahbari NN, Schneider F, et al. Randomized clinical trial on the effect of coffee on postoperative ileus following elective colectomy. Br J Surg 2012; 99: 1530-8.

32. Schmidt J, Stoffels B, Chanthaphavong RS, et al. Differential molecular and cellular immune mechanisms of postoperative and LPS-induced ileus in mice and rats. Cytokine 2012; 59: 49-58.

33. Bellon JM, Manzano L, Larrad A, et al. Endocrine and immune response to injury after open and laparoscopic cholecystectomy. Int Surg 1998; 83: 24-7.

34. Madureira FA, Manso JE, Madureira Filho D, et al. Inflammation in laparoendoscopic single-site surgery versus laparoscopic cholecystectomy. Surg Innov 2013; 21: 263-8.

35. Kang SH, Kim YS, Hong TH, et al. Effects of dexmedetomidine on inflammatory responses in patients undergoing laparoscopic cholecystectomy. Acta Anaesthesiol Scand 2013; 57: 480-7.

Received: 10.03 .2015 , accepted: 12.05 .2015 . 